\title{
Study of laser-induced thermoelastic deformation of native and coagulated ex-vivo bovine liver tissues for estimating their optical and thermomechanical properties
}

\author{
Behrouz Soroushian \\ Ryerson University \\ Department of Physics \\ Toronto, Canada M5B 2K3
}

\author{
William M. Whelan \\ University of Prince Edward Island \\ Department of Physics \\ Charlottetown, Canada C1A 4P3
}

\author{
Michael C. Kolios \\ Ryerson University \\ Department of Physics \\ Toronto, Canada M5B 2K3
}

\begin{abstract}
Several studies have explored the potential of optoacoustic imaging for monitoring thermal therapies, yet the origin of the contrast in the images is not well understood. A technique is required to measure the changes in the optical and thermomechanical properties of tissues upon coagulation to better understand this contrast. An interferometric method is presented for measuring simultaneously the optical and thermomechanical properties of native and coagulated ex-vivo bovine tissue samples based on analysis of the surface displacement of irradiated samples. Surface displacements are measured after irradiation by short laser pulses at $750 \mathrm{~nm}$. A $51 \%$ decrease in the optical attenuation depth is observed for coagulated liver samples compared to native samples. No significant differences in the Grüneisen coefficient are measured in the native and coagulated tissue samples. A mean value of 0.12 for the Grüneisen coefficient is measured for both native and coagulated liver tissues. The displacement profiles exhibit consistent differences between the two tissue types. To assess the changes in the sample mechanical properties, the experimental data also are compared to numerical solutions of the equation for thermoelastic deformation. The results demonstrate that differences in the tissue expansion dynamics arise from higher values of elastic modulus for coagulated liver samples compared to native ones. @ 2010 Society of Photo-Optical Instrumentation Engineers. [DOI: 10.1117/1.3517455]
\end{abstract}

Keywords: thermoelastic expansion; soft tissues; optoacoustic; Gruneisen coefficient; thermomechanical properties

Paper 10367RR received Jun. 29, 2010; revised manuscript received Oct. 5, 2010; accepted for publication Oct. 7, 2010; published online Dec. 2, 2010.

\section{Introduction}

Ablative thermal therapy involves heating a target tissue to temperatures between 50 and $90^{\circ} \mathrm{C}$ to create in-situ coagulation necrosis and cause cell death. This method is considered an effective minimally invasive method to treat solid tumors. Different techniques such as radio-frequency radiation, ${ }^{1}$ high intensity focused ultrasound (HIFU), ${ }^{2}$ or interstitial laser sources ${ }^{3}$ are developed for delivering the thermal energy needed for ablative thermal treatment to a targeted region. An essential part of any successful thermal therapy is a monitoring system, allowing a more accurate assessment of the heated area and to minimize damage to the healthy tissues surrounding the target. Modalities including magnetic resonance temperature imaging, ${ }^{4}$ optical radiance/fluence probes, ${ }^{5}$ and ultrasound imaging ${ }^{6}$ have been investigated for providing such monitoring systems. These methods face challenges such as their cost and patient selectiveness (MRI), imaging depth (IR and optical thermal probes),

Address all correspondence to: Behrouz Soroushian, Bioenginnering Research Center, University of Kansas, 5109 Learned Hall, 1530 West 15th Street, Lawrence, KS 66045-7618. Tel: 785-864-1748; Fax: 785-864-1742. E-mail: bsoroushian@ku.edu. and lack of reliable contrast between lesion and normal tissues (ultrasound imaging).

Optoacoustic imaging (OA) is a new promising modality that recently has been developed and suggested for use in many areas of biomedicine from imaging of breast cancers, ${ }^{7}$ functional and structural imaging of brain, ${ }^{8}$ monitoring of oxygenation to imaging of neovasculation, ${ }^{9}$ and determining blood vessel diameters. ${ }^{10}$ It relies on ultrasonic detection of thermoelastic pressure transients ${ }^{11}$ induced in a target after its irradiation by short laser pulses. Optoacoustic imaging combines the high resolution of ultrasonic imaging with the high tissue contrast of optical imaging techniques. Several studies have shown that the shape and amplitude of optoacoustic signals depend on the optical and thermomechanical properties of the illuminated target. Since thermally induced tissue damage can lead to both changes in optical ${ }^{12,13}$ and mechanical ${ }^{14}$ properties, optoacoustic techniques are suggested for monitoring thermal therapies.

Optoacoustic techniques have been successfully applied to measure tissue temperature during heating and cooling of canine liver, ${ }^{15}$ for monitoring tissue coagulation during thermal therapies, ${ }^{16,17}$ and to assess retinal temperature during its treatment by laser irradiation. ${ }^{18}$ The observed changes in the

1083-3668/2010/15(6)/065002/10/\$25.00@ 2010 SPIE 
optoacoustic signals from thermally treated tissues are so far mainly attributed to the changes in their optical properties. ${ }^{16,19}$ While the changes in the optical properties of thermally treated tissues have been previously studied, there is a paucity of studies that examine the role of changes in the thermal and mechanical properties of tissues and their effect on the optoacoustic signals. In this work, the dynamics of thermoelastic expansion for native and coagulated ex-vivo bovine liver after short pulse laser illumination were studied using an interferometric technique. In the technique proposed, thermomechanical and optical properties of samples were simultaneously assessed at the same sample location.

\section{Theoretical Description}

A short laser pulse that is absorbed by a target creates a small temperature rise. For the short time scales involved in a typical optoacoustic pulsing protocol (typically a few nanoseconds), one can assume that the resulting temperature distribution and the absorbed light distribution are spatially coincident. For the case of a turbid medium like biological tissues or tissue mimicking phantoms, this distribution can be approximated by an axial symmetric function with an exponentially decaying axial profile and a Gaussian radial profile:

$T(r, z)=T_{0} \exp \left(-r^{2} / w^{2}\right) \exp (-z / D)$ and $T_{0}=\frac{\boldsymbol{\Phi} \mu_{a}}{\rho C_{V}}$,

where $C_{V}$ is the heat capacity of the sample at constant volume $\left(\mathrm{J} / \mathrm{m}^{3} \cdot \mathrm{K}\right), \boldsymbol{\Phi}$ is the laser fluence $\left(\mathrm{J} / \mathrm{m}^{2}\right), \rho$ is mass density $(\mathrm{kg})$, and $D$ is the optical attenuation depth (m) (given by the distance along the $z$ axis where laser light intensity diminishes to $1 / \mathrm{e}$ of its value on the surface). This value is defined by the transport theory of light and is given by:

$$
D=\left[3 \mu_{a}\left(\mu_{a}+\mu_{s}^{\prime}\right)\right]^{-1 / 2},
$$

where $\mu_{a}$ and $\mu_{s}^{\prime}$ are the optical absorption $\left(\mathrm{m}^{-1}\right)$ and reduced scattering coefficients $\left(\mathrm{m}^{-1}\right)$ of the material, respectively. A nonuniform temperature distribution [Eq. (1)] leads to the creation of mechanical stresses inside the target and to its thermoelastic deformation. The resulting mechanical wave created after the target thermoelastic deformation can be described by a wave equation in the form of ${ }^{20}$ :

$$
\begin{gathered}
\rho \frac{\partial^{2} \mathbf{u}}{\partial t}-\frac{E}{2(1+\sigma)} \nabla^{2} \mathbf{u}-\frac{E}{2(1+\sigma)(1+2 \sigma)} \nabla(\nabla . \mathbf{u}) \\
=\frac{-E \beta}{3(1-2 \sigma)} \nabla T(r, z),
\end{gathered}
$$

where $\mathbf{u}$ is the displacement vector (m), $E$ is Young's modulus (Pa), $\sigma$ is Poisson's ratio, and $\beta$ is the thermal expansion coefficient of the material $\left(\mathrm{K}^{-1}\right)$. A complete solution of Eq. (3), subjected to a set of the appropriate boundary conditions, is possible through numerical methods. Such a solution (for materials with tissue-like properties) leads to results in which three separate time regimes are distinguishable. ${ }^{21} 1$. A transient regime when internal stresses resulting from nonuniform temperature distribution cause thermoelastic deformation of the illuminated area. In this state the material reconfigures itself to return the net force to zero. 2. A quasi-steady-state regime when the system reaches equilibrium, and although the individual stress components inside the target are not zero, the net force in any direction will be zero. 3. A relaxation phase when stresses decay to zero because thermal dissipation occurs and the temperature distribution in the target becomes uniform. The driving force in the wave in Eq. (3) is the gradient of initial temperature field caused by the light absorption. Depending on the specific properties of the material, the relaxation phase is reached typically a few microseconds after the laser pulse - therefore, the quasi-steadystate regime can last for hundreds of nanoseconds.

Assuming an initial temperature distribution in the form of Eq. (1), it is possible to find an analytical expression for the equilibrium surface displacement on the $z$ axis during the quasisteady-state time regime ${ }^{20}$ :

$$
u_{z}=\frac{2(1+\sigma)}{3} \frac{\Gamma}{\rho c_{L}^{2}} g_{0}(R) \boldsymbol{\Phi},
$$

where $g_{0}(R)$ is a function that describes the optical field in the material and is dependent on the aspect ratio $R$, which is given by the ratio of the laser beam radius $w$ to the optical attenuation depth $D(R=w / D)$. The parameter $\Gamma=c_{L}^{2} \beta / C_{V}$ is the Grüneisen coefficient, an important thermomechanical property of the sample, which in addition to its dependence on the thermal expansion coefficient and the specific heat capacity is also dependent on the longitudinal speed of sound in the target $c_{L}$. This speed can be expressed in terms of the mechanical properties of the sample as:

$$
c_{L}^{2}=\frac{E(1-\sigma)}{\rho(1+\sigma)(1-2 \sigma)} .
$$

Equation (4) shows that measuring the equilibrium displacement of the sample surface after pulsed optical exposure with different laser energies can provide valuable information about the thermal and mechanical properties of the sample. This theoretical framework is used to investigate changes in these properties when ex-vivo bovine liver samples are thermally coagulated.

\section{Materials and Methods}

\subsection{Experimental Setup}

The interferometric setup used to measure the thermoelastic expansion of tissue phantom samples has been described previously. ${ }^{22,23}$ Laser pulses of 6.5-ns duration, $10-\mathrm{Hz}$ repetition rate, and 750-nm wavelength from an optical parametric oscillator (OPO) system (Vibrant B-532, Opotek Incorporated, Carlsbad, California) were directed onto the front surface of tissue phantom samples. The final beam size on the target location was measured using a photographic sheet and had an elliptical cross section with major and minor axes of 5 and $3 \mathrm{~mm}$, respectively. A part of the pump beam was directed onto a fast silicon photodiode (DET10A, 200 to $1100 \mathrm{~nm}, 1$-ns rise time, Thorlabs, Newton, New Jersey) to measure the pulse energy and also to synchronize the data acquisition and the incident pump pulse. The incident angle of the pump beam onto the sample was approximately $36 \mathrm{deg}$. According to Snell's law, this yields an angle of refraction of $26 \mathrm{deg}$ into the tissue based on indices of refraction of 1.00 for air and 1.40 for tissue.

The thermoelastic movements of the sample's surface were measured using a modified Michelson interferometer. It is built around a cw solid-state laser (CrystaLaser GCL025-S, CrystaLaser, Reno, Nevada) operating at a wavelength of $532 \mathrm{~nm}$. The laser beam was split into two arms 


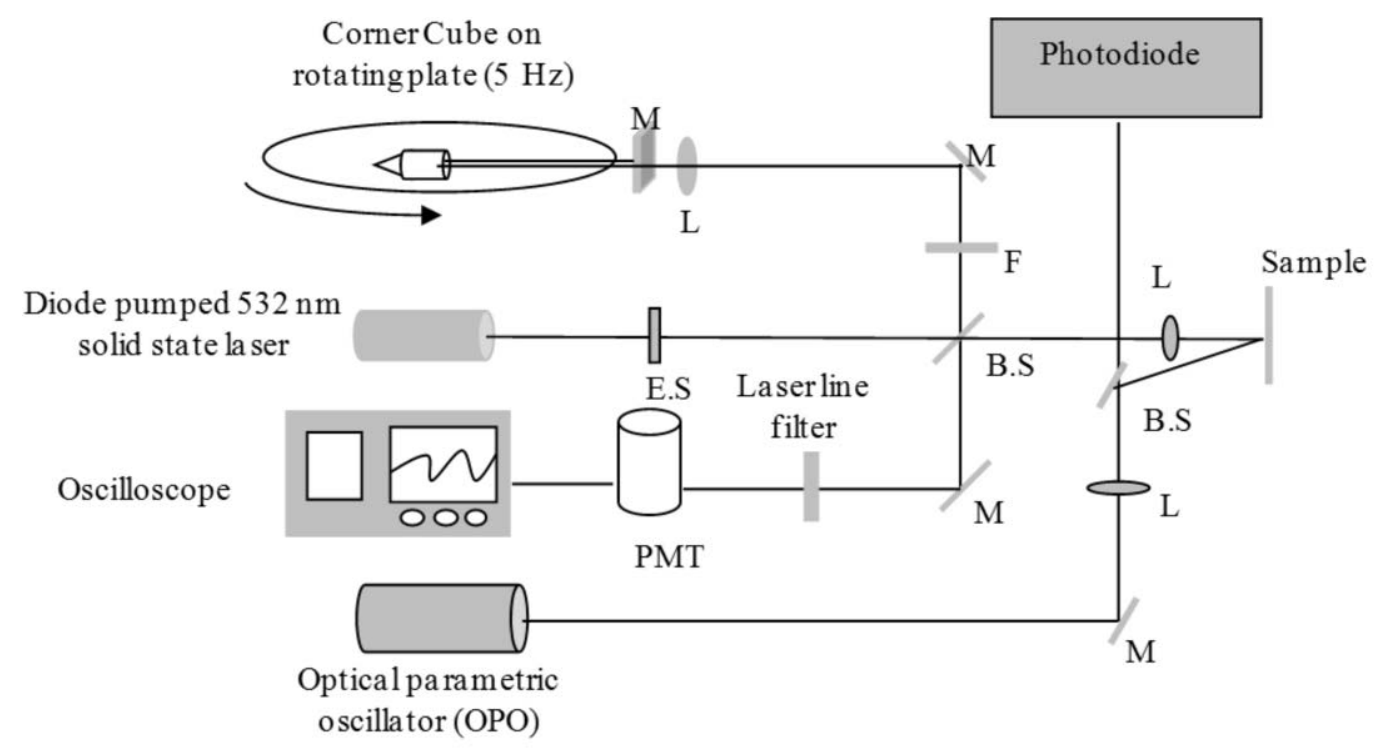

M: mirror, L: lens, B.S: Beam Splitter, F: Filter, E.S: Electronic Shutter, PMT: Photomultiplier

Fig. 1 Schematic diagram of the experimental interferometry setup.

with one arm directed toward a corner cube mounted on a rotating table, permitting a constant modulation on the interference pattern when the target surface is not moving. This allowed the improvement of spatial and temporal resolution of the interferometry setup beyond those of a conventional Michelson interferometer, and also provided the capability of detecting the direction of the target surface movement. During and after OPO illumination, the resulting interference signals were detected by a photomultiplier (Hamamatsu H7732P-11, Hamamatsu City, Japan), amplified, and then digitized on an oscilloscope (Agilent DSO6054A, $500 \mathrm{MHz}$, Agilent, Santa Clara, California) for analysis. A schematic diagram of the experimental interferometry setup is shown in Fig. 1.

\subsection{Sample Preparation}

Seven samples of bovine liver (each on a different day) were purchased from a local market. The samples remained sealed in their packaging and kept at $4{ }^{\circ} \mathrm{C}$ to avoid gassing and slow tissue degradation. From each piece of liver, two samples of about $5 \times 5 \times 1 \mathrm{~cm}$ were prepared. One of the samples was placed in a $70^{\circ} \mathrm{C}$ water bath for $30 \mathrm{~min}$. The heated samples were then removed and cut in smaller rectangular pieces with dimensions of approximately 1 to $2 \mathrm{~cm}$. The untreated liver sample was also cut in smaller pieces with dimensions similar to the heated one. The accuracy of our interferometry technique is improved for tissue samples with a smooth, flat surface. As these two surface qualities can be affected by the heating process, smaller pieces were cut from the heated samples, yielding better surface uniformity. One piece from each sample was placed in the center of two identical aluminum cylindrical containers. Gelatin (type A, Sigma Life Science, Saint Louis, Missouri) was dissolved in hot water and cooled down to around $40^{\circ} \mathrm{C}$ before it was poured around the samples in the containers. The containers then were placed in a refrigerator at a temperature of $6^{\circ} \mathrm{C}$ to let the gelatin harden and fix the samples in place. After solidification of the gelatin, the front face of the tissue sample, which was exposed to air, was used for the interferometric experimental measurements.

\subsection{Data Analysis}

The interference pattern detected by the photomultiplier was collected for a period of $10 \mu \mathrm{s}$, with the pump laser pulses firing $2 \mu \mathrm{s}$ after the beginning of each acquisition frame. The acquired data were exported to Matlab (The Mathworks, Natick, Massachusetts) for analysis. Displacement of the target surface was calculated using the Hilbert transform on the interferogram. ${ }^{24}$ Signals from a silicon photodiode detecting a portion of the pump laser pulse were used to estimate energy of each laser pulse hitting the target surface. The measurements were calibrated prior to the experiments by placing an accurate laser power meter (Ophir Nova II meter and PE25-SH measuring head, Ophir Optronics Limited, Jerusalem, Israel) at the target location, and comparing the readings from it with data obtained from the silicon photodiode. The signal from the photodiode follows the laser pulse, and so its duration is much faster than the rate of change in the interference fringe pattern. Both signals were digitized and recorded on the same oscilloscope and within the same acquisition time frame of $10 \mu \mathrm{s}$. A low sampling rate for digitizing the two signals can lead to inaccuracy in the measurement of laser pulse energy, while a high sampling rate increases the size of data files recorded by the oscilloscope. Therefore, a moderate digitization sample rate of $500 \mathrm{MS} / \mathrm{s}$ was selected to reduce discretization of the readings for the laser pulse energies and at the same time keep the size of data files reasonable.

\subsection{Numerical Calculation}

The thermoelastic wave in Eq. (3) was numerically solved by using the finite-difference time domain (FDTD ${ }^{25}$ method in a cylindrical coordinate system. To further simplify the problem, 


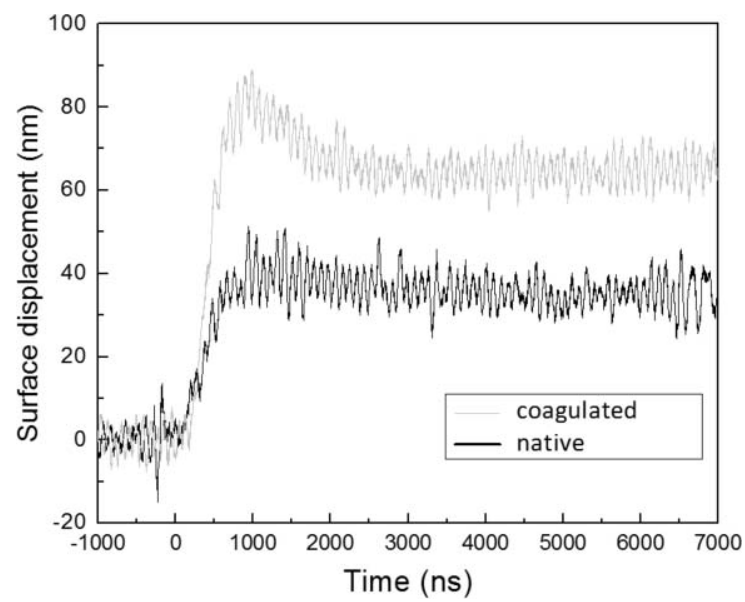

Fig. 2 Sample surface displacement traces obtained for coagulated and native ex-vivo liver tissue samples after their irradiation by short laser pulses at $750 \mathrm{~nm}$ (pulse fired at time $t=0 \mathrm{~ns}$ ).

it is assumed that temperature field induced by laser pulse in the sample is axially symmetric. A staggered mesh was employed to discretize the space for the two remaining dimensions of the coordinate system ( $z$ and $r$ ), with the $z$ axis set to be along the irradiation direction. Only half of the space along the $r$ axis is discretized to reduce the required computational memory. The grid size is chosen to be smaller than one-tenth of the optical attenuation depth in the sample (grid size is $8.5 \times 8.5 \mu \mathrm{m}$ ). Then, the temporal step size is determined to satisfy the Courant condition for solution stability. ${ }^{26}$ For the interface between air and the sample surface, a free boundary condition (i.e., pressure at $z=0$ is zero) is applied. The axial symmetry of the problem requires that the radial component of displacement at the center of the cylinder $(r=0)$ also be zero. An absorbing boundary layer on the two outer edges with rigid constraints (displacement at the edges is equal to zero) is used to eliminate artificial reflections of the deformation wave from these edges. ${ }^{27}$

\section{Results and Discussion}

\subsection{Dynamics of Thermoelastic Deformation}

\subsubsection{Optical attenuation depth}

Typical sample surface displacement profiles for native and coagulated ex-vivo bovine livers after their irradiation by a short laser pulse at $750 \mathrm{~nm}$ are shown in Fig. 2. Thermoelastic stresses are generated within the sample. In response to these stresses, the sample surface expands and reaches its maximum; the time it takes to reach this maximum is characterized by the optical attenuation depth and speed of sound in the sample. An approximation for slope of initial rise in the surface displacement curve is given by ${ }^{28}$ :

$$
u(t)=u_{0}\left[1-\exp \left(-c_{L} t / D\right)\right],
$$

where $\mathrm{u}_{0}$ is the maximum expansion of the target after laser irradiation and is determined by thermomechanical properties of the sample, $c_{L}$ is the longitudinal speed of sound in the material, and $D$ is the optical attenuation depth for a specific light wavelength. Previous studies have shown that the difference between the propagation speed of sound in the native and coagulated tissues at the same temperature is not statistically significant. ${ }^{29}$ Therefore, one can assume that the sound speed in both native and coagulated tissue samples is similar. The optical attenuation depth in native and coagulated ex-vivo bovine livers at room temperature (for a wavelength of $750 \mathrm{~nm}$ ) was estimated assuming a longitudinal speed of sound equal to $1500 \mathrm{~m} / \mathrm{s}$, and using Eq. (6) for the initial rise in the surface displacements. The values are corrected for the propagation angle of light into the sample by dividing them by $\cos (26 \mathrm{deg}) .{ }^{28}$ The results are presented in Fig. 3, and in all cases except one, show decrease in the optical attenuation depth for the coagulated liver samples compared to the noncoagulated ones. The behavior observed in the one exceptional case is likely due to local tissue heterogeneity, where the native element of this sample pair had the smallest optical attenuation depth among all native samples tested. In the case of native liver samples, the measured optical attenuation depth varies between 556 and $1270 \mu \mathrm{m}$ with a mean value of $860 \pm 197 \mu \mathrm{m}$ (mean $\pm 99 \%$ confidence limit). This value for the coagulated liver samples is between 392 and $694 \mu \mathrm{m}$ with a mean of $421 \pm 66 \mu \mathrm{m}$ (mean $\pm 99 \%$ confidence limit). The variation observed among native samples is likely due to some variability in tissue degradation prior to purchase. These postmortem changes including, for example, tissue dehydration, ${ }^{30,31}$ can affect the optical and thermomechanical properties of samples. The results show an average decrease of $51 \%$ in optical attenuation depth of the coagulated tissue samples compared to the untreated ones. A two sample paired t-test reveals that the difference between the values for the optical attenuation depth of the two sample groups is statistically significant within the 5\% level $(t=3.43$ and $P=0.014)$. Using another method, Ritz et al. found a $68 \%$ decrease of the optical attenuation depth at $830 \mathrm{~nm}$ for the coagulated porcine liver samples compared to native ones. ${ }^{13}$ Larin, Larina, and Esenaliev measured a decrease of $45 \%$ for the optical attenuation depth at $1064 \mathrm{~nm}$ for coagulated canine liver compared to the native samples. ${ }^{16}$ The differences between measured values of the optical attenuation depth in literature may be due to the different methods employed

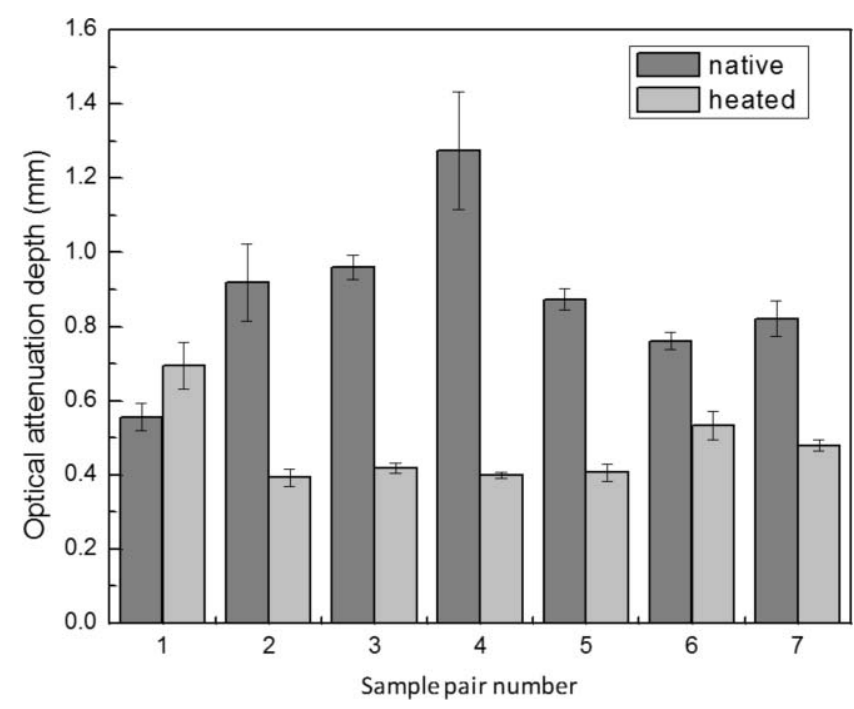

Fig. 3 Measured optical attenuation depth of native and coagulated exvivo bovine liver samples irradiated at $750 \mathrm{~nm}$ (mean value \pm standard error of the mean). 
Table 1 Optical attenuation depth of ex-vivo liver tissue in different species as reported in the literature.

\begin{tabular}{lcccc} 
Species & $\begin{array}{c}\text { Wavelength } \\
(\mathrm{nm})\end{array}$ & $\begin{array}{c}\text { Native sample } \\
(\mu \mathrm{m})\end{array}$ & $\begin{array}{c}\text { Coagulated } \\
\text { sample }(\mu \mathrm{m})\end{array}$ & Reference \\
\hline Porcine liver & 830 & $3130 \pm 50$ & $990 \pm 90$ & {$[13]$} \\
Canine liver & 1064 & $1343 \pm 163$ & $556 \pm 173$ & {$[16]$} \\
Rat liver & 633 & 612 & & {$[42]$} \\
Bovine liver & 633 & 789 & & {$[43]$} \\
Bovine liver & 750 & $860 \pm 197$ & $421 \pm 66$ & this work \\
Human liver & 670 & $750 \pm 70$ & & {$[34]$} \\
\hline
\end{tabular}

for the measurements, data analysis, and sample preparation. By using time-resolved detection of laser-induced transient stresses in tissue, Oraevsky, Jacques, and Tittel reported optical attenuation depths of 360 and $5000 \mu \mathrm{m}$ for native bovine liver at wavelengths of 532 and $1064 \mathrm{~nm}$, respectively. ${ }^{11}$ The values of optical attenuation depth for liver tissues obtained in some previous studies are summarized in Table 1.

\subsubsection{Grüneisen coefficient}

After an initial expansion, the sample surface may contract before reaching a new equilibrium displacement. In our experiments, prominent contractions were generally observed in the case of coagulated samples, while in the case of uncoagulated samples such contractions were considerably smaller or nonexistent (Fig. 2). In the latter case, one can assume that equilibrium and maximum displacement of the sample surface are equivalent. For assessment of the changes occurring in thermal and mechanical properties of tissue samples after coagulation, we compared the equilibrium displacement of the sample surface

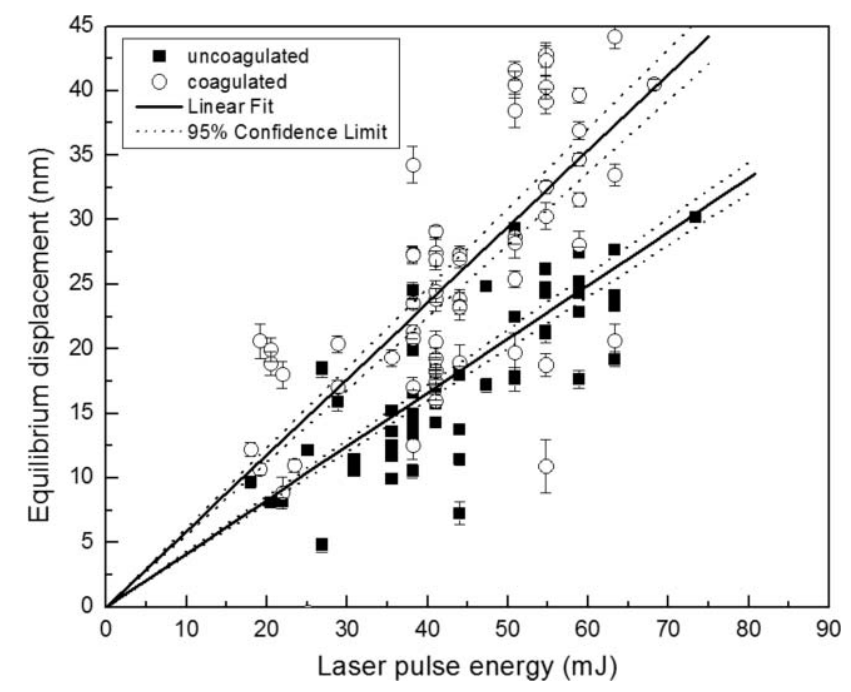

Fig. 4 Equilibrium displacement of native (empty circles) and coagulated (filled squares) liver samples versus laser pulse energy for two liver samples. Linear regressions and 95\% confidence limits are given for both samples.

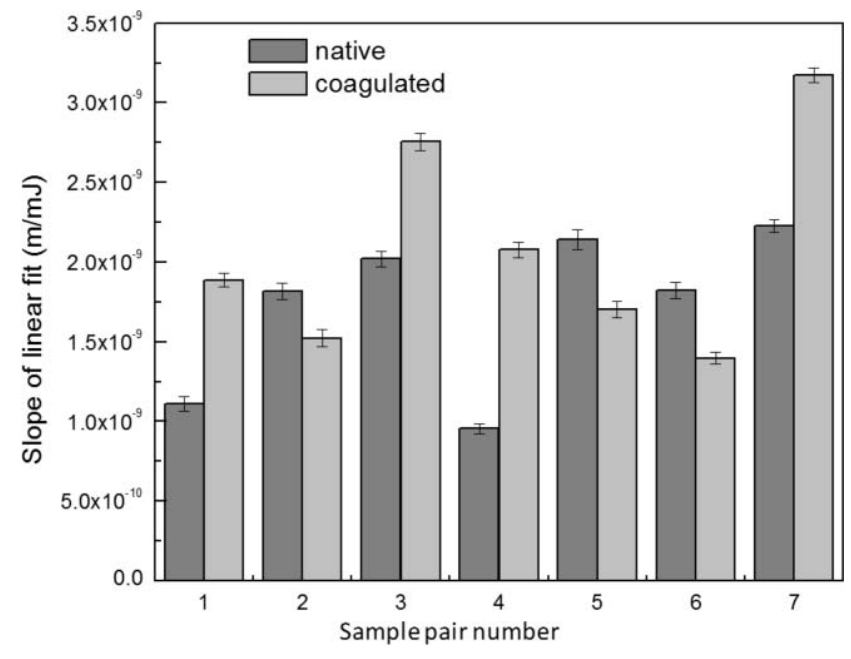

Fig. 5 Slopes of linear fits for the equilibrium displacement of native and coagulated liver samples versus laser pulse energies. Error bars represent the standard deviation of the measured surface displacement.

after its initial expansion. For each signal, the time-resolved displacement of the sample surface was smoothed by averaging over neighboring points. The resulting displacements between 4000 and $5500 \mathrm{~ns}$ after the firing of the laser pulse were averaged and their standard deviation was calculated. These values were considered equilibrium displacements of the sample surface.

The equilibrium displacement of the sample surface was plotted against the energy of incident laser pulses to measure the Grüneisen coefficient. We considered a linear fit passing through the origin (no displacement for a laser pulse with energy of zero, see Eq. (4)). An example of fitted data for a pair of native and coagulated samples is shown in Fig. 4. The correlation coefficients of the linear regressions range from 0.78 to 0.92 . The results show an average of $19.6 \%$ increase in slope of the fitted lines for the coagulated samples compared to the native tissues. However, a two sample paired t-test reveals that the difference between values of slope for the native and coagulate liver tissues is significant only at $P=0.24(t=-1.31)$ level. Both a decrease and increase in slope of the linear fit for the coagulated liver tissues compared to native samples are observed. A bar chart of slopes for the linear regressions is presented in Fig. 5. Since sound speeds in the native and coagulated liver samples at room temperature are similar, ${ }^{29}$ the changes of the slope cannot be explained by difference of the sound speed in the samples. The geometrical correction factor $\mathrm{g}_{0}(R)$ for each sample is calculated using the mean values of the optical attenuation depth (measured as described in the previous section) and assuming a laser beam radius of $2.5 \mathrm{~mm}$ on the targeted tissues. These values of $\mathrm{g}_{0}(R)$ are then inserted into Eq. (4) to estimate values of the Grünesien coefficient for the native and coagulated liver samples. The result is presented as a bar chart in Fig. 6. The Grünesien coefficient for the seven native samples ranges from 0.071 to 0.16 , while this parameter for the coagulated samples varies between 0.088 and 0.2 . A similar variation is previously reported for values of the Grünesien coefficient for knee meniscus. ${ }^{23}$ The average value of the Grünesien coefficient for both groups of the native and coagulated liver samples is 0.12 . Considering the relation of the Grüneisen coefficient 


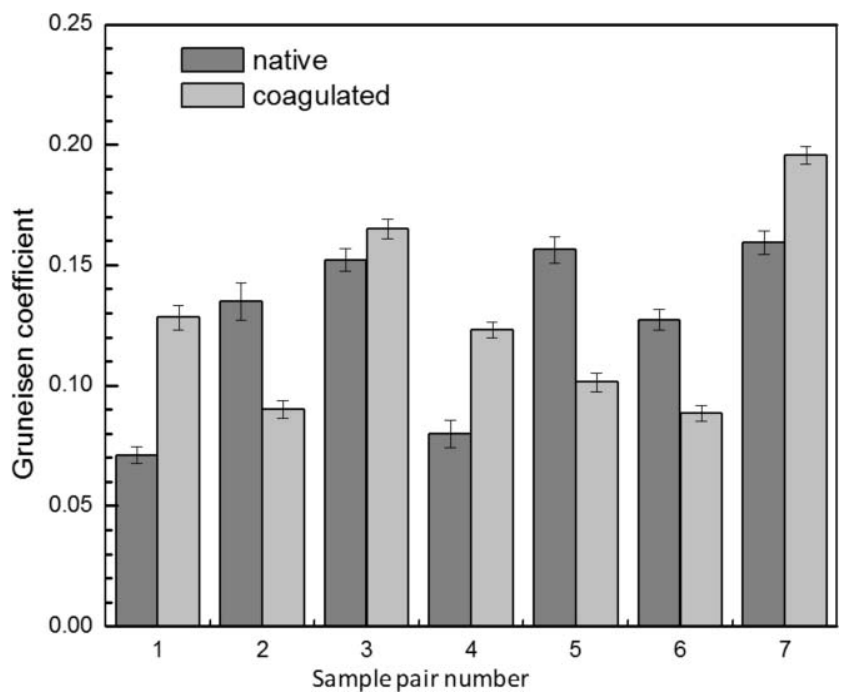

Fig. 6 Estimated values of the Grüneisen coefficient for native and coagulated liver samples, using the average values of the optical attenuation depth and the slope of the linear regression to the equilibrium surface displacements, versus laser pulse energies for each sample. Error bars are propagations of the uncertainties related to the optical attenuation depths and the slope of the linear fit.

with the physical properties of tissue (since $\Gamma=c_{L}^{2} \beta / C_{V}$ ), and using reported values of these properties for liver tissues $\left[\beta=3 \times 10^{-4} \mathrm{~K}^{-1}, C_{V}=3600 \mathrm{~J} \cdot \mathrm{kg}^{-1} \cdot \mathrm{K}^{-1}\right.$ and $c_{L}=1500$ $\mathrm{m} / \mathrm{s}],{ }^{32,33}$ one calculates $\Gamma=0.19$, which is consistent with our results. However, the sensitivity of the current measurements to noise, in addition to natural variability of the biological samples (e.g., variations in fat and connective tissue content in the small liver areas irradiated) prevents detection of any significant difference between Grüneisen coefficients in the two groups of samples $(t=-0.088, P=0.93)$.

Surface displacements of tissue samples are measured along the optical axis of the sample arm in the interferometry setup. It is known that an important source of uncertainty in measuring the surface displacement is alignment of the target surface relative to this axis. ${ }^{44}$ Since soft tissues and especially liver samples are usually prone to large deformations under relatively small forces ${ }^{35}$ environmental mechanical noise causes changes in the alignment of the tissue surface along the optical axis of the interferometer. There is also a considerable fluctuation in spatial profiles of the laser pulses, and the tissue sample in the irradiated area $\left(47 \mathrm{~mm}^{2}\right)$ is heterogeneous. This leads to variation of the induced heat profile in the sample when it moves because of mechanical noise. As an example, for laser pulses with the same energy, a typical maximum standard deviation of 36\% for scattered measurements of the quasi-steady-state displacement was obtained.

\subsubsection{Mechanical properties and elastic modulus}

The dynamics of the thermoelastic expansion of the sample's surface for the two groups of native and coagulated liver tissue samples are significantly different: a more prominent retraction after the initial expansion of the tissue surface was observed in the case of coagulated liver samples. Even for the signals in which displacement in the quasi-steady-state was the same

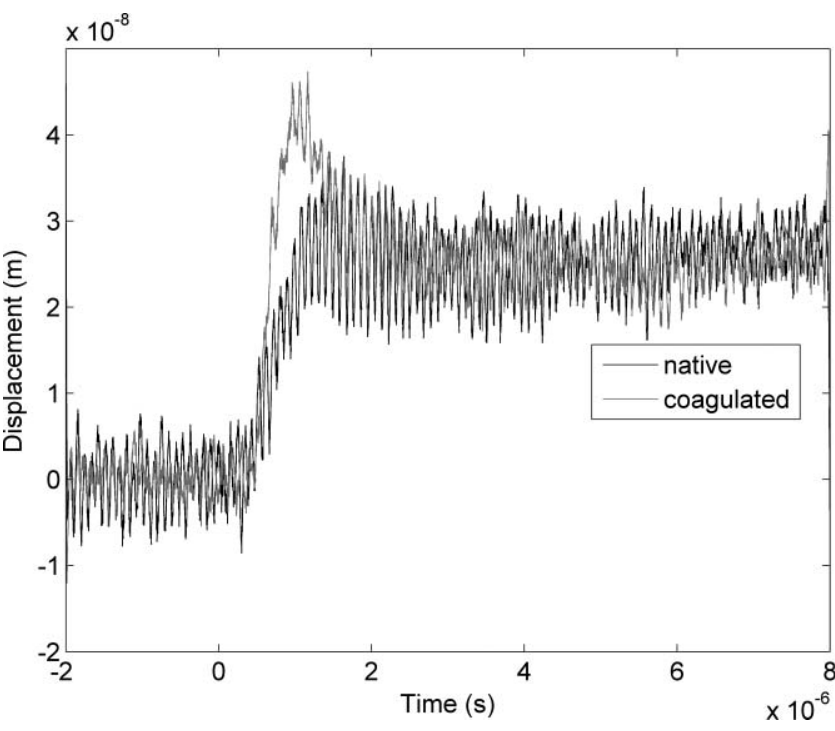

Fig. 7 Sample surface displacement traces obtained for coagulated and native ex-vivo liver tissue samples after their irradiation by short laser pulses at $750 \mathrm{~nm}$. The surfaces attain a same displacement in quasi-steady-state, but the coagulated sample shows more pronounced retraction after its initial expansion.

value, the coagulated sample showed a more pronounced retraction after its initial expansion compared to the native sample. Such behavior is shown in Fig. 7. To quantify this effect, a single exponential decay function in the form of:

$$
d(t)=d_{0}+a \cdot \exp (-t / \tau),
$$

in which the parameter $\tau$ is a measure of the characteristic time for the surface retraction, was fitted, wherever possible, to the displacement signals after the sample surface attained its maximum displacement. For some samples, the surface retraction in the displacement signal is nonexistent. In these cases, the fitting was not performed and such signals (less than 5\% of all signals for the uncoagulated samples and only in the rare cases of signals for the coagulated samples) were excluded from the analysis. Geometric averages of the measured retraction rates $\tau$ for each tissue sample were calculated. For giving a measure of variance for these values, the $95 \%$ confidence interval of the values is computed. Then the antilog of both limits is obtained to get the confidence interval of the average values. ${ }^{36}$ Note that the measure of variation is not symmetrical around the mean values themselves. The results are presented as a bar chart in Fig. 8 . A significant difference $(t=5.4$ and $P<0.005)$ was observed between results of the native and coagulated tissue samples. The average values of the characteristic retraction times for native and coagulated samples are $1.77 \pm 0.59$ (mean $\pm 99 \%$ confidence limit) and $0.7 \pm 0.15 \mu$ s (mean $\pm 99 \%$ confidence limit), respectively. Fitting the experimental data with a single exponential decay function was easier in the case of the coagulated tissues, and the resulting retraction times were spread over smaller ranges of values, while in comparison with the native tissue samples, the larger standard deviations indicate a weaker goodness of fit.

The magnitude of the surface retraction [parameter $a$ in Eq. (7)] is another parameter that can be used to demonstrate the differences in the expansion dynamics between two groups of 
Soroushian, Whelan, and Kolios: Study of laser-induced thermoelastic deformation of native and coagulated. . .

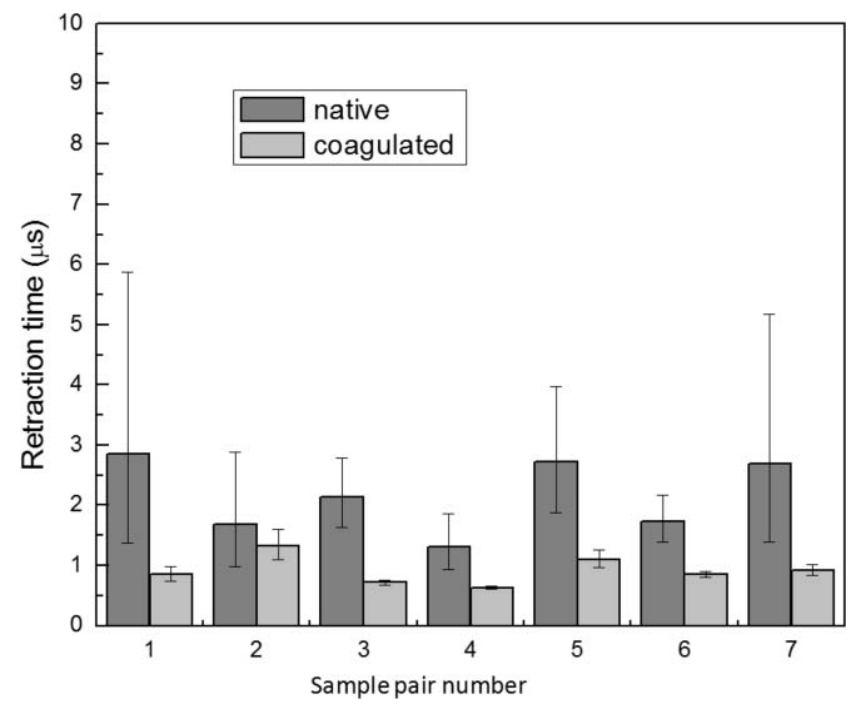

Fig. 8 Characteristic surface retraction time after its initial expansion in native and coagulated liver samples. For explanation on the error bars, see Sec. 4.1.3.

the native and coagulated tissue samples. Relative magnitudes of sample surface retractions to the maximum displacements were calculated and their averages are shown as a bar chart in Fig. 9. Again, a significant difference $(t=-3.76, P<0.01)$ between the retraction magnitudes of the native and coagulated tissue surfaces is measured. The native tissue samples show an average retraction of $19 \%$ relative to their maximum displacements, while this value for the coagulated tissues on average is $30 \%$. To relate this behavior of the tissue samples with their optical and thermomechanical properties, thermoelastic deformation of the tissue samples after their irradiation by laser pulses is studied using numerical solution of Eq. (3).

\subsection{Thermoelastic Deformation of Tissues}

Numerical solutions of the equation of thermoelastic expansion are obtained using the method previously described in Sec. 3.4.

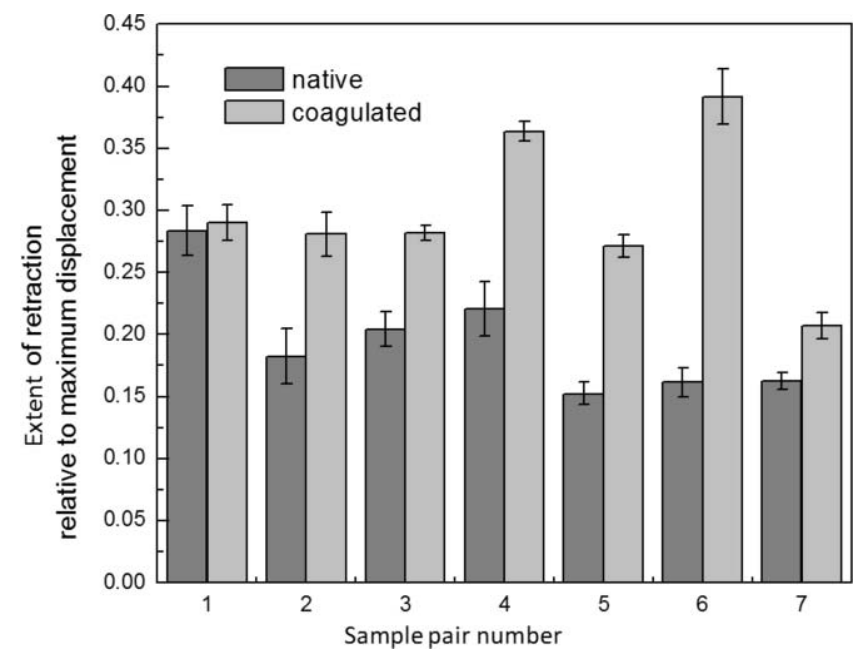

Fig. 9 Extent of surface retraction relative to its maximum displacement for native and coagulated liver samples (mean value \pm standard error of the mean).
The physical properties of tissues were varied to simulate thermoelastic deformations observed through our experiments. The numerical solutions are not used in an iterative way to find the best fit for the experimental data, since it requires excessive computation times. Parameters of Eq. (3) are set in such a way that the longitudinal speed of sound remains $1500 \mathrm{~m} / \mathrm{s}$ in all cases. This requires modifying Poisson's ratio when the elastic modulus is modified [see Eq. (5)]. However, the necessary variations of Poisson's ratio are limited to a very small range of values around 0.5 , which is expected for mostly incompressible soft tissues. ${ }^{37}$

The geometrical properties of the laser-induced heat profile in the target can be determined by using the method previously described in Sec. 4.1.1. For the sake of simplicity, the cross section of the pump laser beam on the target was assumed circular with a radius of $2500 \mu \mathrm{m}$. The optical attenuation depth for the typical signals presented in Fig. 1 was calculated by fitting the initial rise to a function in the form of Eq. (6) (560 and $300 \mu \mathrm{m}$ for the native and coagulated sample, respectively). Also, a thermal expansion coefficient of $5 \times 10^{-4} \mathrm{~K}^{-1}$ for both samples was assumed. Using these values, the numerical solution of thermoelastic expansion of samples with different values of elastic modulus was computed. Then, these solutions of the surface displacement after their initial rises were fitted to a function in the form of Eq. (7). The characteristic retraction times and the extent of the surface retraction were compared to those values for the typical experimental signals shown in Fig. 2. These comparisons for the characteristic retraction time and extent of surface retraction are presented in Figs. 10 and 11, respectively.

Using a value of $25 \mathrm{kPa}$ for the elastic modulus and peak temperature rise $\left[T_{0}\right.$ in Eq. (3)] of $0.16^{\circ} \mathrm{C}$, the numerical solution to the surface displacement agrees with the experimentally measured data for the native tissue. On the other hand,

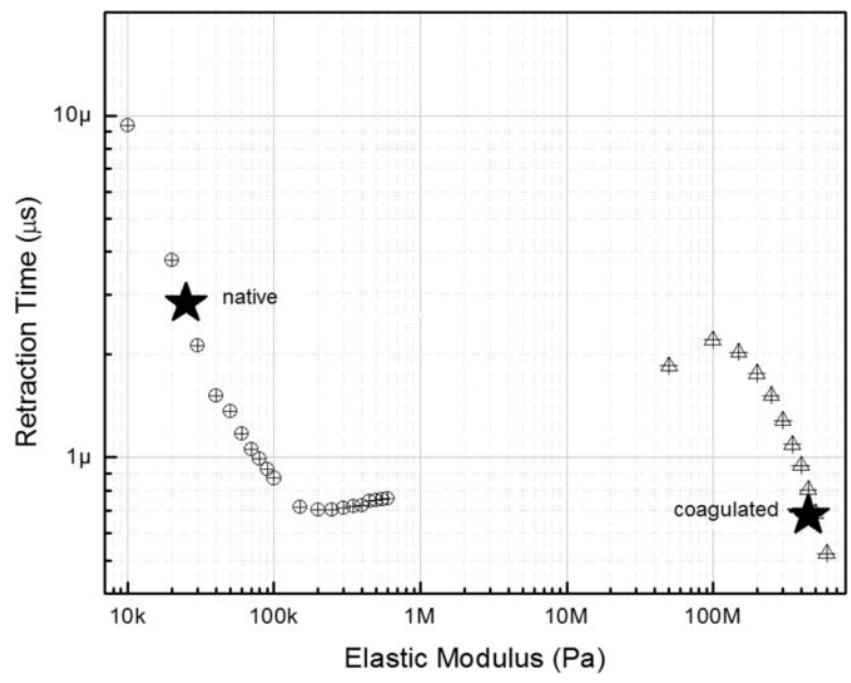

Fig. 10 Characteristic surface retraction times predicted by numerical solutions of the wave equation for different values of elastic modulus. Thermal expansion coefficient is assumed to be $\beta=5 \times 10^{-5} \mathrm{~K}^{-1}$ and the laser beam radius $w=2500 \mu \mathrm{m}$. The numerical solutions are computed for to two sets of parameters corresponding to two signals presented in Fig. 1 (circles): $D=560 \mu \mathrm{m}, T_{0}=0.16^{\circ} \mathrm{C}$, and (triangles): $D=300 \mu \mathrm{m}$ and $T_{O}=0.62^{\circ} \mathrm{C}$. The obtained values for the two experimental signals are shown with black stars. 


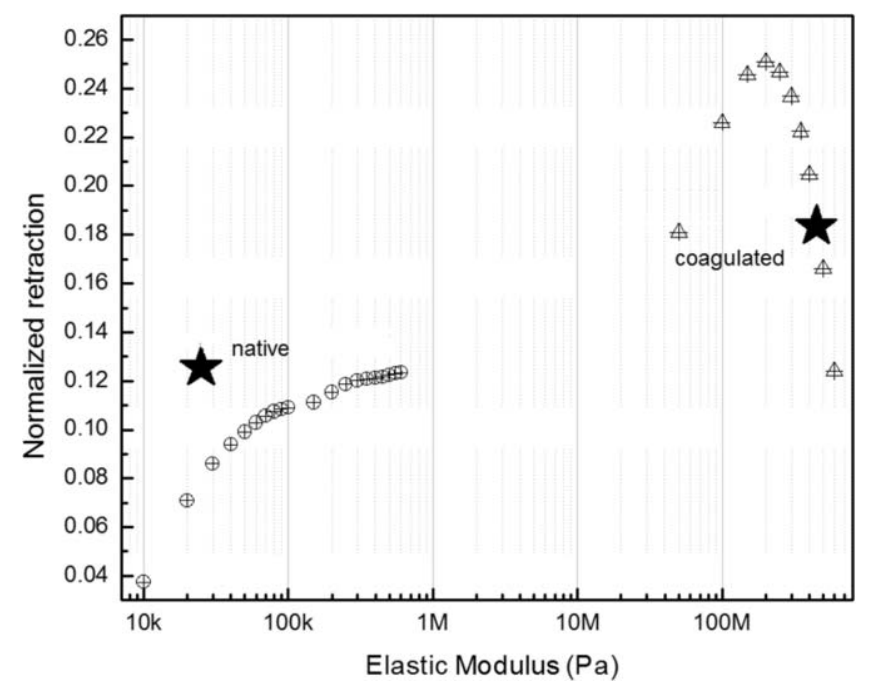

Fig. 11 Extent of the surface retraction relative to its maximum displacement predicted by numerical solutions of the wave equation for different values of elastic modulus. Thermal expansion coefficient is assumed to be $\beta=5 \times 10^{-5} \mathrm{~K}^{-1}$ and the laser beam radius $w=$ $2500 \mu \mathrm{m}$. The numerical solutions are computed for two set of parameters corresponding to two signals presented in Fig. 1 (circles): $D=560 \mu \mathrm{m}, T_{0}=0.16^{\circ} \mathrm{C}$, and (triangles): $D=300 \mu \mathrm{m}$ and $T_{0}$ $=0.62^{\circ} \mathrm{C}$. The obtained values for the two experimental signals are shown with black stars.

the experimental signal for the coagulated tissue generates a better match to the theoretical curve when assuming a value of $450 \mathrm{MPa}$ for the elastic modulus and a peak temperature rise of $0.62^{\circ} \mathrm{C}$. Surface displacements calculated using these values are compared against the experimental signals in Fig. 12. The disparity between the extent of the surface retraction relative to its maximum displacement measured experimentally, and the numerical solution prediction for the native liver sample can be attributed to the fact that the experimental signal has noise at higher frequencies, and that when the retraction time is very long, the quality of fit between the measured signal and Eq. (7) is worse. The numerical calculations are not used for finding a best fit to the experimental data. Only a few sets of parameters are examined to find the agreement between experimental data and predictions of the numerical solutions, using, however, accepted values for the physical constants used in the equations. The prominent retraction of the sample surface after expansion can be attributed to greater values of the elastic modulus of the coagulated liver tissue samples compared to native ones. Other studies also have shown stiffening and increase of elastic modulus of tissue samples following coagulation. ${ }^{14,38,39}$

Elastic moduli that are estimated in the current study are higher than the values reported in the other studies. This can be attributed to the fact that these references deal with the estimation of the tissue's mechanical properties at lower loading frequencies. For example, Kiss, Varghese, and Hall performed experiments over a frequency range from 0.1 to $400 \mathrm{~Hz},{ }^{14}$ and reported values of 2 and $27 \mathrm{kPa}$ for the elastic modulus of native and coagulated ex-vivo canine liver samples, respectively. It is known that the elastic modulus of tissue samples is frequency dependent and increases with higher frequencies. ${ }^{40}$ In other studies with higher values of mechanical pressure and greater deformations of the native tissue target, values for the elastic modulus ranging from $110 \mathrm{kPa}$ to $1.02 \mathrm{MPa}$ were measured. ${ }^{41}$ By studying the second derivative of the surface displacement, which is a measure of acceleration and hence the forces on the target surface, one can estimate the rate by which the thermal stresses in the tissue sample are working. The second derivative of the typical signals shown in Fig. 2 are calculated and shown in Fig. 13. It shows that peak-to-peak changes in surface stresses are of the order of 770 and $820 \mathrm{~ns}$ for these two signals. These variations correspond to frequencies higher than $1 \mathrm{MHz}$ and therefore the measurement is probing moduli at these timescales. On the other hand, in our experiments the deformation of the target tissue samples due to these stresses is very small (of the order of nanometers). To the best of our knowledge, the current work is among very few experiments that provide measurements on deformation of soft tissues at high frequencies and low deformations.
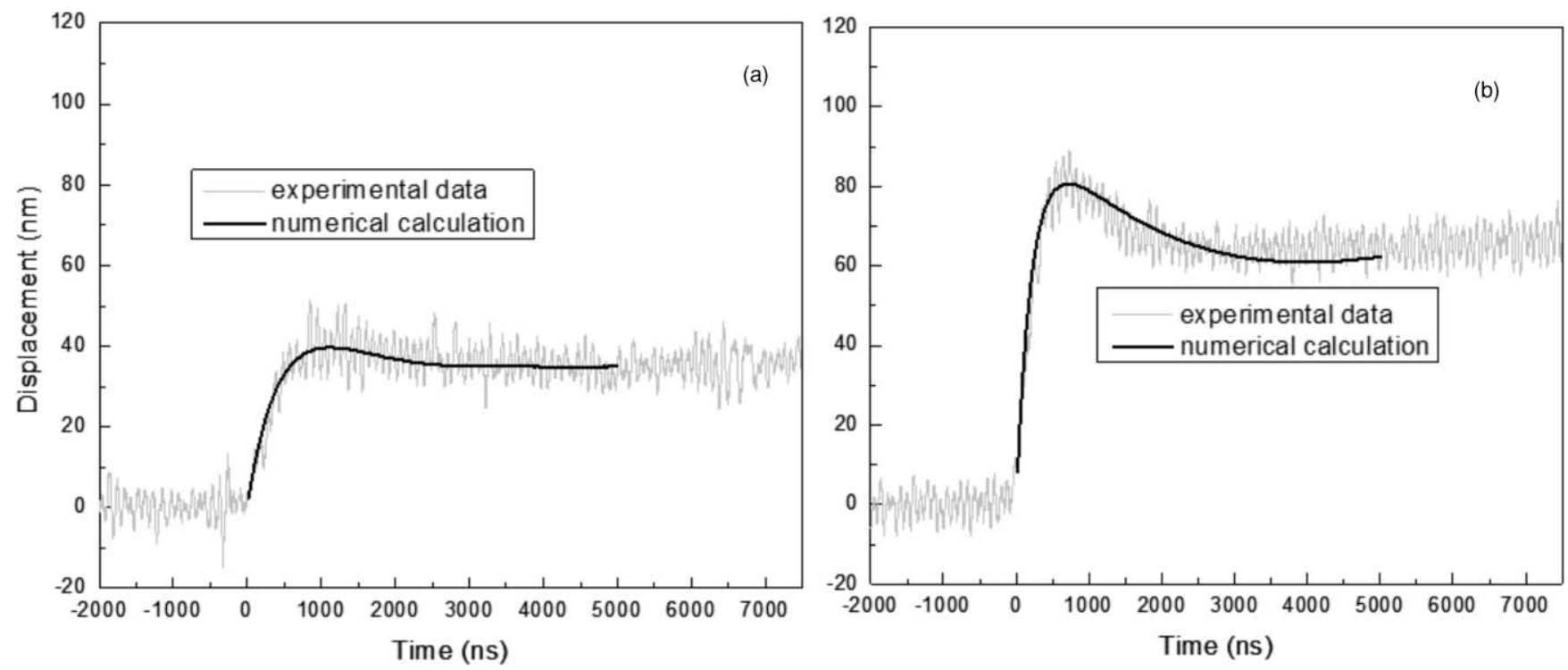

Fig. 12 Predictions of the numerical solutions for the thermoelastic expansions of targets agree with the experimental signals presented in Fig. 1. 


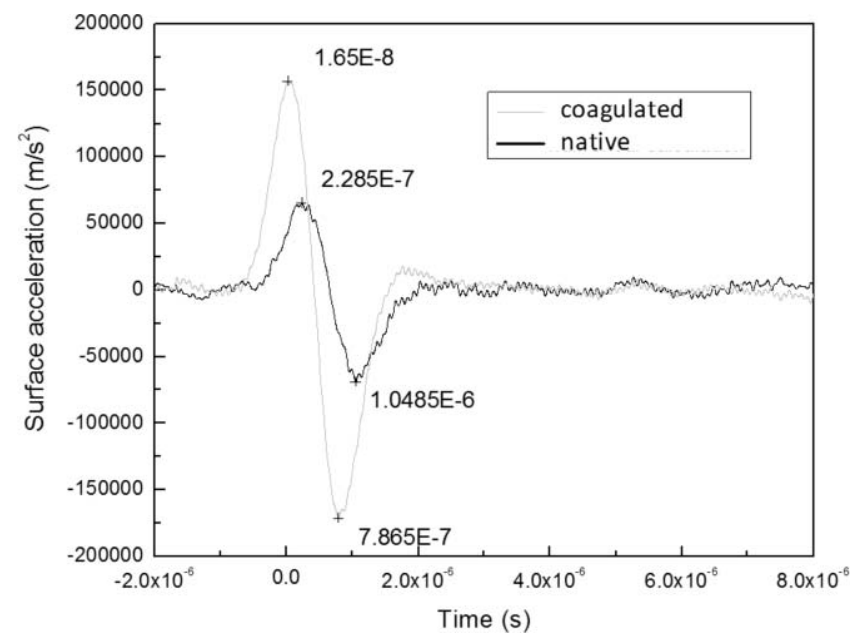

Fig. 13 Second derivative of surface displacement for the experimental signals presented in Fig. 1.

\section{Conclusion}

The study of thermoelastic deformation of tissue samples after incidents of short laser pulses on them can provide a wealth of information about their optical and thermomechanical properties. Interferometric measurements are performed to study these types of deformations in native and coagulated ex-vivo bovine liver samples. A key advantage of this technique is that from the same dataset, both optical and thermomechnical properties can be derived. Therefore, the property measurements are both spatially and temporally coincident, something that cannot be achieved easily with other techniques. A decrease of the optical attenuation depth for the coagulated liver samples compared to native ones is observed. One can estimate the Grüneisen coefficient of tissue samples by using the slope of linear fit to the equilibrium displacement of the target surface as a function of laser pulse energy. The natural variability of the biological samples (e.g., variations in fat and connective tissue content in the small liver areas irradiated) and sensitivity of our measurements to noise prevented us from finding a significant difference in the Grüneisen coefficient between the two groups. However, dynamics of thermoelastic deformations for the two groups are distinctively different. The surface of the coagulated tissue samples shows a more pronounced retraction after its initial expansion compared to native liver samples. Comparison of the experimental data with the numerical solution of a thermoelastic wave equation show that this behavior can be explained assuming an increase in the elastic modulus of the coagulated tissue samples compared to the native one. The results suggest that optoacoustic imaging can be used for guidance and monitoring of thermal therapies by exploiting the changes that in the optical and thermomechanical properties of tissues occur while they undergo thermal treatment.

\section{Acknowledgment}

The authors would like to gratefully acknowledge Arthur Worthington for his assistance in the experiments. Financial support was provided by the Atlantic Innovation Fund, the Natural Sciences and Engineering Research Council of Canada, the Canadian Institutes of Health Research (grant CHRPJ323745-
60), and the Canada Foundation for Innovation (CFI). This research was undertaken, in part, thanks to funding from the Canada Research Chairs Program awarded to Michael Kolios.

\section{References}

1. A. N. Mirza, B. D. Fornage, N. Sneige, H. M. Kuerer, L. A. Newman, F. C. Ames, and S. E. Singletary, "Radiofrequency ablation of solid tumors," Cancer J. 7(2), 95-102 (2001).

2. J. E. Kennedy, G. R. TerHaar, and D. Cranston, "High intensity focused ultrasound: surgery of the future," Br. J. Radiol. 76, 590-599 (2003).

3. T. J. Vogl, R. Straub, K. Eichler, O. Sollner, and M. G. Mack, "Colorectal carcinoma metastases in liver: laser-induced interstitial thermotherapylocal tumor control rate and survival data," Radiol. 230(2), 450-458 (2004).

4. B. Quesson, J. A. de Zwart, and C. T. W. Moonen, "Magnetic resonance temperature imaging for guidance of thermotherapy," J. Magn. Reson. Imag. 12(4), 525-533 (2000).

5. L. Chin, M. Pop, W. Whelan, M. Sherar, and A. Vitkin, "Optical method using fluence or radiance measurements to monitor thermal therapy," Rev. Sci. Instrum. 74(1), 393-395 (2003).

6. S. Siebers, U. Scheipers, C. Welp, J. Werner, and H. Ermert, "A classification system for monitoring thermal therapies," Ultrasonics Symp. IEEE 2, 1126-1129 (2005).

7. S. A. Ermilov, T. Khamapirad, A. Conjusteau, M. H. Leonard, R. Lacewell, K. Mehta, T. Miller, and A. A. Oraevsky, "Laser optoacoustic imaging system for detection of breast cancer," J. Biomed. Opt. 14(2), 024007 (2009).

8. X. Wang, Y. Pang, G. Ku, X. Xie, G. Stoica, and L.V. Wang, "Noninvasive laser-induced photoacoustic tomography for structural and functional in vivo imaging of the brain," Nature Biotechnol. 21(7), 803-806 (2003).

9. R. I. Siphanto, K. K. Thumma, R. G. M. Kolkman, T. G. van Leeuwen, F. F. M. de Mul, J. W. van Neck, L. N. A. van Adrichem, and W. Steenbergen, "Serial noninvasive photoacoustic imaging of neovascularization in tumor angiogenesis," Opt. Express 13(1), 89-95 (2004).

10. R. G. M. Kolkman, J. H. G. M. Klaessens, E. Hondebrink, J. C. W. Hopman, F. F. M. de Mul, W. Steenbergen, J. M. Thijssen, and T. G. van Leeuwen, "Photoacoustic determination of blood vessel diameter," Phys. Med. Biol. 49, 4745-4756 (2004).

11. A. A. Oraevsky, S. L. Jacques, and F. K. Tittel, "Measurement of tissue optical properties by time-resolved detection of laser-induced transient stress," Appl. Opt. 36(1), 402-415 (1997).

12. C.-T. Germer, A. Roggan, J. P. Ritz, C. Isbert, D. Albrecht, G. Müller, and H. J. Buhr, "Optical properties of native and coagulated human liver tissue and liver metastases in the near infrared range," Lasers Surg. Med. 23(4), 194-203 (1998).

13. J. P. Ritz, A. Roggan, C. Isbert, G. Mller, H. J. Buhr, and C. T. Germer, "Optical properties of native and coagulated porcine liver tissue between 400 and 2400 nm," Lasers Surg. Med. 29(3), 205-212 (2001).

14. M. Z. Kiss, T. Varghese, and T. J. Hall, "Viscoelastic characterization of in vitro canine tissue," Phys. Med. Biol. 49(18), 4207-4218 (2004).

15. I. V. Larina, K. V. Larin, and R. O. Esenaliev, "Real-time optoacoustic monitoring of temperature in tissues," J. Phys. D: Appl. Phys. 38(15), 2633-2639 (2005).

16. K. V. Larin, I. V. Larina, and R. O. Esenaliev, "Monitoring of tissue coagulation during thermotherapy using optoacoustic technique," $J$. Phys. D: Appl. Phys. 38(15), 2645-2653 (2005).

17. C. Richter, G. Spirou, A. A. Oraevsky, W. M. Whelan, and M. C. Kolios, "Examination of contrast mechanisms in optoacoustic imaging of thermal lesions," Proc. SPIE 6086, 60861K (2006).

18. J. Kandulla, H. Elsner, R. Birngruber, and R. Brinkmann, "Noninvasive optoacoustic online retinal temperature determination during continuous-wave laser irradiation," J. Biomed. Opt. 11(4), 041111 (2006).

19. R. F. Castelino, W. M. Whelan, and M. C. Kolios, "Photoacoustic detection of protein coagulation in albumen-based phantoms," Proc. SPIE 6856, 685626 (2008).

20. D. Albagli, M. Dark, C. Von Rosenberg, L. Perelman, I. Itzkan, and M. S. Feld, "Laser-induced thermoelastic deformation: a three- 
dimensional solution and its application to the ablation of biological tissue," Med. Phys. 21(8), 1323-1331 (1994).

21. I. Itzkan, D. Albagli, M. L. Dark, L. T. Perelman, C. Von Rosenberg, and M. S. Feld, "The thermoelastic basis of short pulsed laser ablation of biological tissue," Proc. Natl. Acad. Sci. USA 92, 1960-1964 (1995).

22. B. Soroushian, W. Whelan, and M. Kolios, "Assessment of optomechanical behavior of biological samples by interferometry," Proc. SPIE 7177, 71771X-6 (2009)

23. M. L. Dark, L. T. Perelman, I. Itzkan, J. L. Schaffer, and M. S. Feld, "Physical properties of hydrated tissue determined by surface interferometry of laser-induced thermoelastic deformation," Phys. Med. Biol. 45, 529-539 (2000).

24. J. P. Harkin and D. A. Flavin, "Interferometric displacement tracking based on Hilbert transform processing," Proc. SPIE 4204, 89-98 (2001).

25. D. H. Huang, C. K. Liao, C. W. Wei, and P. C. Li, "Simulations of optoacoustic wave propagation in light-absorbing media using a finitedifference time-domain method," J. Acoust. Soc. Am. 117(5), 27952801 (2005).

26. C. T. Schröder and W. R. Scott, Jr., "On the stability of the FDTD algorithm for elastic media at a material interface," IEEE Trans. Geosci. Remote Sens. 40(2), 474-481 (2002).

27. X. B. Tian, I. B. Kang, G. Y. Kim, and H. S. Zhang, "An improvement in the absorbing boundary technique for numerical simulation of elastic wave propagation," J. Geophys. Eng. 5(2), 203-209 (2008).

28. B. P. Payne, V. Venugopalan, B. B. Mikic, and N. S. Nishioka, "Optoacoustic determination of optical attenuation depth using interferometric detection," J. Biomed. Opt. 8(2), 264-272 (2003).

29. U. Techavipoo, T. Varghese, Q. Chen, T. A. Stiles, J. A. Zagzebski, and G. R. Frank, "Temperature dependence of ultrasonic propagation speed and attenuation in excised canine liver tissue measured using transmitted and reflected pulses," J. Acoust. Soc. Am. 115(6), 2859-2865 (2004).

30. D. Zhu, Q. Luo, and J. Cen, "Effects of dehydration on the optical properties of in vitro porcine liver," Laser Surg. Med. 33, 226-231 (2003).

31. M. P. Otternsmeyer, A. E. Kedok, R. D. Howe, and S. L. Dawson, "The effects of testing environment on the viscoelastic properties of soft tissues," Int. Symp. Med. Sim. Springer-Verlag, Berth (2004).

32. NCRP, "Report No. 113: exposure criteria for medical diagnostic ultrasound: I. Criteria based on thermal mechanisms Report No. 113," National Council on Radiation Protection and Measurements,, p. 53, Bethesda, MD (1992)
33. K. Giering, I. Lamprecht, O. Minet, and A. Handke, "Determination of the specific heat capacity of healthy and tumorous human tissue," ThermochimicaActa 251(C), 199-205 (1995).

34. S. Nakamura, Y. Nishiwaki, S. Suzuki, S. Sakaguchi, Y. Yamashita, and K. Ohta, "Light attenuation of human liver and hepatic tumors after surgical resection," Lasers Surg. Med. 10(1), 12-15 (1990).

35. N. G. Shrive, Soft tissue strain measurement," Optical Measurement Methods in Biomechanics, pp. 156-172, J. C. Shelton and J. F. Orr, Eds., Chapman and Hall, London (1997).

36. J. Olivier, W. D. Johnson, and G. D. Marshall, "The logarithmic transformation and the geometric mean in reporting experimental IgE results: what are they and when and why to use them? Ann. Allergy, Asthma Immunol. 100(4), 333-337 (2008)

37. C. Chui, E. Kobayashi, X. Chen, T. Hisada, and I. Sakuma, "Combined compression and elongation experiments and non-linear modelling of liver tissue for surgical simulation," Med. Biol. Eng. Computi. 42(6), 787-798 (2004).

38. S. Bharat, U. Techavipoo, M. Z. Kiss, W. Liu, and T. Varghese, "Monitoring stiffness changes in lesions after radiofrequency ablation at different temperatures and durations of ablation," Ultrasound Med. Biol. 31(3), 415-422 (2005).

39. M. Zhang, B. Castaneda, J. Christensen, W. Saad, K. Bylund, K. Hoyt, J. G. Strang, J. G. Strang, D. J. Rubens, and K. J. Parker, "Real-time sonoelastography of hepatic thermal lesions in a swine model," Med. Phys. 35(9), 4132-4141 (2008).

40. D. Valtorta and E. Mazza, "Dynamic measurement of soft tissue viscoelastic properties with a torsional resonator device," Med. Image Anal. 9(5 SPEC. ISS.), 481-490 (2005).

41. H. Saraf, K. T. Ramesh, A. M. Lennon, A. C. Merkle, and J. C. Roberts, "Mechanical properties of soft human tissues under dynamic loading," J. Biomechan. 40(9), 1960-1967 (2007).

42. P. Parsa, S. L. Jacques, and N. S. Nishioka, "Optical properties of rat liver between 350 and $2200 \mathrm{~nm}, "$ Appl. Opt. 28(12), 2325-2330 (1989).

43. T. J. Pfefer, L. S. Matchette, C. L. Bennett, J. A. Gall, J. N. Wilke, A. J. Durkin, and M. N. Ediger, "Reflectance-based determination of optical properties in highly attenuating tissue," J. Biomed. Opt. 8(2), 206-215 (2003).

44. P. G. Charette, I. W. Hunter, and P. J. Hunter, "Large deformation mechanical testing of biological membranesusing speckle interferometry in transmission. I: experimental apparatus," Appl. Opt. 36(10), 2238 2245 (1997). 